\title{
Hepatocellular carcinoma, novel therapies on the horizon
}

\author{
Imane El Dika ${ }^{1,2}$, Iman Makki ${ }^{3}$, Ghassan K. Abou-Alfa ${ }^{1,2}$ \\ ${ }^{1}$ Memorial Sloan Kettering Cancer Center, New York, NY, USA; ${ }^{2}$ Weill Medical College at Cornell University, New York, NY, USA; ${ }^{3}$ Icahn School \\ of Medicine Mount Sinai St. Luke's West, New York, NY, USA \\ Contributions: (I) Conception and design: All authors; (II) Administrative support: I El Dika, GK Abou-Alfa; (III) Provision of study materials or \\ patients: None; (IV) Collection and assembly of data: All authors; (V) Data analysis and interpretation: I El Dika, GK Abou-Alfa; (VI) Manuscript \\ writing: All authors; (VII) Final approval of manuscript: All authors. \\ Correspondence to: Ghassan K. Abou-Alfa, MD, MBA. Memorial Sloan Kettering Cancer Center, 300 East 66th street, New York, NY, 10065, USA. \\ Email: abou-alg@mskcc.org.
}

\begin{abstract}
Hepatocellular carcinoma (HCC) is the most common primary liver cancer and is associated with high mortality rate. Incidence remains high due to the persistent prevalence of viral hepatitis, alcoholic cirrhosis, and non-alcoholic fatty liver disease (NFLD). Despite screening efforts, the majority of patients present with advanced disease, add to the high risk of recurrence after curative surgery. Conventional chemotherapy did not alter the nature history of advanced and metastatic HCC. The discovery of multiple tyrosine kinase inhibitors (TKIs) led to the approval of sorafenib as first efficacious therapy. A new era in the treatment paradigm of HCC is evolving. Since the advent of sorafenib as an active treatment option for patients presenting with advanced or metastatic disease, several agents have been examined. This was linked with many failures, and success stories to celebrate. Herein, we describe the historical progress and current advances of systemic therapies post-sorafenib. Lenvatinib, regorafenib, cabozantinib, ramucirumab, pembrolizumab, and nivolumab, are all presently added and available therapeutic options in the advanced setting. The evaluation of novel treatment combinations including anti-angiogenic, TKIs plus checkpoint inhibitors, add to dual checkpoint inhibitors is evolving rapidly starting with the advent of the combination of atezolizumab plus bevacizumab. Combining local and systemic therapies is being actively investigated, as an option for locally advanced disease conventionally treated with locoregional approaches. The horizon remains promising and continues to evolve for HCC a disease long considered with unmet needs.
\end{abstract}

Keywords: Hepatocellular carcinoma (HCC); tyrosine kinase inhibitors (TKIs); immunotherapy

Submitted Mar 08, 2020. Accepted for publication May 21, 2020.

doi: $10.21037 /$ cco-20-113

View this article at: http://dx.doi.org/10.21037/cco-20-113

\section{Introduction}

Primary liver cancer has become the second cause of cancer related mortality globally, with hepatocellular carcinoma (HCC) accounting for $90 \%$ of primary liver cancers $(1,2)$. HCC with a high incidence in Asia, and Africa $(3,4)$, is now on the rise in Europe and the United States likely secondary to the increase in hepatitis $\mathrm{C}$ and non-alcoholic fatty liver disease and non-alcoholic steatohepatitis (NFLD/ NASH) (5-8). Early stage HCC can potentially be cured through surgical resection, ablation, or liver transplantation; however, recurrence rates remain high and survival remains low (9-13). The majority of patients are diagnosed at a later more advanced stage that lacks any curative options (14). Historically, systemic therapy had failed to show any mortality benefit in advanced HCC patients until sorafenib was approved by the United States Food and Drug Administration (FDA) (15) followed by approval worldwide. Several agents demonstrated clinical activity since. Tyrosine kinase inhibitors (TKIs) showed survival benefit with tolerable side effects, yet limited improvement in prognosis and HCC remains an area of unmet need (2). In the TKI era, overall survival in patients with advanced HCC was averaging around ten months (16), highlighting 
the importance of further effort. Experimenting with checkpoint inhibitors alone and in combination with other modalities and novel therapeutic targets followed. Herein, we will review current systemic therapies and expand upon some of the relevant ongoing trials and future horizons.

\section{TKIs}

\section{Sorafenib}

Sorafenib, an inhibitor of multiple receptor tyrosine kinases that include Raf kinase, vascular endothelial growth factor receptor (VEGFR) and other kinases, was the first to show a survival benefit in HCC. The SHARP trial concluded that the median overall survival reached 10.7 months in the sorafenib group compared to 7.9 months with placebo [hazard ratio (HR) in the sorafenib group, 0.69; $95 \%$ confidence interval (CI), 0.55 to $0.87 ; \mathrm{P}<0.001]$ (16). Since then, sorafenib became the standard of care in management of advanced HCC worldwide $(15,17)$.

\section{Lenvatinib}

A period of time that spanned over almost a decade elapsed since the approval of sorafenib, during which multiple efforts did not demonstrate any improvement in outcomes compared to sorafenib in advanced HCC (18-20). Lenvatinib is a multiple receptor kinase inhibitor mostly affecting angiogenesis through inhibiting VEGFR 1-3, fibroblast growth factor receptor (FGFR 1-4), platelet growth factor receptor alpha (PEDGFR), among other kinases (21). The REFLECT trial evaluated the noninferiority of lenvatinib versus sorafenib as a first line therapy for advanced HCC. The study showed that patients randomized to the lenvatinib group had a median survival of 13.6 months and a progression free survival (PFS) of 7.4 months compared to 12.3 and 3.7 months, respectively, in the sorafenib group. Objective response rate (ORR) as defined by modified RECIST (mRECIST) was $24.1 \%$ (20.2-27.9\%) for lenvatinib compared to $9.2 \%$ (6.6$11.8 \%)$ in the sorafenib group, $\mathrm{P}<0.0001$. The difference was consistently noted when using masked independent imaging review according to RECIST1.1 (18.8\% vs. $6.5 \%$, $\mathrm{P}<0.0001)$ (22). The REFLECT study led to the approval of lenvatinib as another frontline therapy in advanced HCC.

Though both sorafenib and lenvatinib have overlapping targets, it appears that lenvatinib could be a more potent inhibitor of VEGFR (21). Other than the approved second line regorafenib (23) discussed next, that would require prior tolerance and progression on sorafenib, other second line therapy studies required prior sorafenib by default as it was the sole approved systemic therapy at that time. A post hoc analysis from the phase 3 REFLECT trial looked at OS of patients on subsequent cancer therapies. Patients who were randomized to initial lenvatinib had an OS of $20.8 \mathrm{vs}$. 17 months for sorafenib-treated patients (HR 0.87; 95\% CI, 0.67-1.14) (24). This adds to the safety profiles of both agents where experience may favor sorafenib while more tolerant and more manageable adverse events may favor lenvatinib.

\section{Regorafenib}

Regorafenib, an oral TKI, was evaluated as a second line treatment for patients who have radiologically progressed on and tolerated sorafenib in the first line. In the RESORCE study (23), patients receiving regorafenib had an improvement in median OS to 10.6 months (95\% CI, 9.112.1) versus 7.8 months (6.3-8.8) for patients who received placebo In an exploratory analysis of the RESORCE trial, median OS on sequential sorafenib and regorafenib therapy (from initiation of sorafenib until death) reached 26 months (22.6-28.1) for patients who received regorafenib in the second line compared to 19.2 months (16.3-22.8) for placebo (25).

\section{Cabozantinib}

Cabozantinib is another oral TKI with potent angiogenesis inhibition in addition to suppressive activity against AXL and MET, both involved in tumor growth and metastasis (26). The CELESTIAL trial randomized patients with no more than two prior lines of therapy one of which is sorafenib, to cabozantinib versus placebo (27). The study showed an improvement in overall survival in favor of cabozantinib versus placebo. Patients treated with cabozantinib had a median OS of 10.2 months whereas median OS with placebo was 8 months (HR, 0.76; 95\% CI, 0.63 to 0.92; $\mathrm{P}=0.005)$. Median PFS was superior for cabozantinib over placebo, 5.2 vs. 1.9 months (HR, 0.44; $95 \%$ CI, 0.36 to 0.52 ; $\mathrm{P}<0.001)$. Cabozantinib also had an acceptable and tolerable side effect profile. The occurrence of adverse events such as hypertension and palmar-plantar erythrodysesthesia was associated with improved outcomes (28). The positive outcomes were irrespective of patients' age (29) or albuminbilirubin (ALBI) grade (30). ALBI score uses albumin and 
bilirubin levels, two objective readily available biomarkers, to evaluate the severity of liver dysfunction and predict the prognosis of patients with acute on chronic liver failure. High bilirubin levels combined with low albumin predict a worse outcome in patients with HCC (31). It permits further stratification of liver function within the Child-Pugh score and provides a reliable predictive score in patients with HCC $(32,33)$. Compared to Child-Pugh, ALBI have suggested a better prognostic tool in specific scenarios (34), yet may need further validation.

\section{Anti-angiogenics}

\section{Bevacizumab}

Bevacizumab inhibits angiogenesis by directly binding circulating VEGF and preventing it from acting on its receptors. Bevacizumab has been studied as a potential agent in treating HCC alone or in combination with first line sorafenib. A phase II pilot study was conducted that assessed bevacizumab's efficacy and tolerability in patients with HCC after disease progression on sorafenib (35). The study showed that at a 12 -month median followup, the average time to symptomatic progression was 3.8 months, time to radiological progression was 3.9 months, and OS was 9.5 months. The patients tolerated the drug well, one patient with already known grade 3 esophageal varices experienced a grade 3 esophageal bleeding. A limitation of this study is the small number of patients enrolled (12 patients). Another phase I/II trial studied the use of bevacizumab in combination with sorafenib as first line therapy in patients with locally advanced or metastatic HCC. In the phase II part of the trial more than $50 \%$ of the patients enrolled exhibited grade 3 toxicities and no significant efficacy endpoints were noted which led to study discontinuation (36).

\section{Ramucirumab}

Ramucirumab is an IgG-1 recombinant monoclonal antibody that inhibits VEGF-2 receptor and was tested as a potential therapeutic agent in treatment of advanced HCC. REACH trial is a phase III trial conducted to evaluate the safety and efficacy of ramucirumab in patients whose disease has progressed on sorafenib (37). Though ramucirumab demonstrated a tolerable safety profile, the effect on median survival [9.2 months in the treatment group (95\% CI, 8.010.6) vs. 7.6 months in the placebo group (95\% CI, 6.0-9.3)] was not statistically significant. A prespecified stratification based on $\alpha$-fetoprotein (AFP) level showed an improvement OS from 4.2 to 7.8 months in patients with AFP of $400 \mathrm{ng} / \mathrm{mL}$ or greater. These findings led to the subsequent phase III trial (REACH-2), of second line ramucirumab in patients with advanced HCC and elevated AFP concentrations of $400 \mathrm{ng} / \mathrm{mL}$ or higher (38). The study showed an improved median OS of 8.5 vs. 7.3 months in the placebo group as well as improved PFS of 2.8 vs. 1.6 months. Ramucirumab demonstrated a tolerable safety profile and can be an added option for patients with elevated AFP of $400 \mathrm{ng} / \mathrm{mL}$ or higher and have progressed on sorafenib.

\section{Immunotherapy}

The unique vascular anatomy and physiologic role of the liver make it vastly exposed to endogenous and exogenous antigens, giving the liver its description as "immunologically tolerant" organ (39). This feature may halt the development of anticancer immunity. The liver microenvironment is enriched by growth factors which act on dendritic cells to contribute to this tolerogenic profile (40). Furthermore, HCC secondary to hepatitis infection exhibit an exhausted $T$ cell phenotype where immune cells might not be able to mount a response against tumor cells (40). PD-L1 is highly expressed in HCC and in the peritumoral antigen presenting cells (APCs) $(41,42)$. Interestingly APCs express the ligands for CTLA-4 and PD-1 and blockage of these pathways should play an important role in the liver where priming of T-cells is usually limited (43). In a retrospective study, the presence of low regulatory $\mathrm{T}$ cells (Tregs) and high CD8 $\mathrm{T}$ cells was associated with decreased rate of recurrence and improved survival in HCC (44). Interestingly, cases of spontaneous tumor regression were reported (45), suggesting that there is a possibility to overcome the liver immunosuppressive abilities. Preclinical and early stage clinical trials suggested potential signals of efficacy for immune checkpoint blockade in HCC leading to the exploration of immune checkpoint blockade against various targets.

\section{Nivolumab}

Nivolumab is a programmed death-1 (PD-1) receptor inhibitor. A phase II study CheckMate 040 showed that patients receiving nivolumab had a durable ORR of $20 \%$ and a relatively good safety profile (46). According to the study, response to therapy lasted at least 6 months 
in $91 \%$ of responders and at least 12 months in $55 \%$ of responders. Nivolumab was granted accelerated FDA approval as $2^{\text {nd }}$ line treatment in advanced HCC based on results of this trial. A randomized, multicenter, phase III study CheckMate 459 followed, comparing nivolumab to sorafenib as first-line treatment. Nivolumab treated patients had a median OS of 16.4 months versus 14.7 months for the sorafenib treated patients (HR, 0.85; 95\% CI, 0.72-1.02; $\mathrm{P}=0.0752$ ). Expectantly, the nivolumab treated group compared to sorafenib group exhibited a higher ORR $15 \%$ vs. $7 \%$ respectively, and a more favorable safety profile with $22 \%$ grade 3-4 adverse events related to treatment vs. $49 \%$ respectively (47).

\section{Pembrolizumab}

Pembrolizumab is another PD-1 receptor inhibitor that was evaluated in phase II clinical trial for patients with advanced HCC after first line therapy (Keynote-224). The study showed median PFS of 4.8 months (95\% CI, 3.4 to 6.6), with 6-month PFS and OS rates were $43.1 \%$ and $77.9 \%$, respectively. Disease control rate was 62\% (95\% CI, $52-71 \%$ ), with $77 \%$ of responders showing a duration of response (DOR) of 9 months; the median DOR was not reached (3.1-14.6+ months) (48). Pembrolizumab was granted an accelerated approval by the FDA as second line treatment in advanced HCC. The KEYNOTE-240 trial followed. A phase III trial of pembrolizumab versus placebo; 413 patients were randomized. The study did not show an improvement in OS. Median OS was 13.9 months (95\% CI, 11.6-16.0 months) in the pembrolizumab investigational arm and 10.6 months (95\% CI, 8.3-13.5 months) for placebo (HR, 0.78; one sided $\mathrm{P}=0.0238$ ).PFS was not significantly improvement with pembrolizumab, where it reached 3 months versus 2.8 months with placebo (HR, 0.78; one sided $\mathrm{P}=0.0209$ ) (49), raising the same argument for the limited benefit if at all of checkpoint inhibitors single agent in patients with advanced HCC.

\section{Combination therapies}

Despite all tremendous efforts, single agent therapies in HCC were not able to bypass the previously suggested ceiling of median OS of less than one year (50), prompting the development of new therapeutic notions. Combination therapies have already been evaluated extensively and in different variations mainly anti-VEGF plus checkpoint inhibitors, TKI plus checkpoint inhibitors, and combinations of checkpoint inhibitors.

Blockade of VEGF/VEGFR axis could likely potentiate the effects of immunomodulators such as PD-1/PDL1 inhibitors (51). Studies have shown that inhibiting angiogenesis induces hypoxia in tumor tissue, which in turn leads to upregulation of immune checkpoint protein PD-L1 (52). In addition, studies have also revealed that inhibiting VEGF in itself may improve tumor-specific T-cell activity (53). This placed antiangiogenic agents as attractive candidates for use in combination with anti-PD-1/antiPD-L1 agents (51). Below we describe the combination of checkpoint inhibitors with antiangiogenic agents, TKIs, and other checkpoint inhibitors. Selected ongoing trials testing this hypothesis are summarized in Table 1.

\section{Anti-VEGF plus checkpoint inbibitors}

Vascular endothelium plays direct role in antigen recognition through interaction between endothelium and immune cells $(54,55)$. Recently, combining immunotherapy and anti-angiogenesis has shown clinical efficacy in different tumors such as lung (56) and genitourinary cancers (57). One rationale behind this combination is that proangiogenic factors have immunosuppressive activity (58), and by inhibiting angiogenesis, immune checkpoint blockade can be potentiated. There is growing evidence of potential synergy between both pathways and preclinical data showing better antitumor effect across various cancer models (59).

\section{Atezolizumab and bevacizumab}

The combination of the PDL-1 inhibitor atezolizumab plus bevacizumab was initially looked at in a phase $1 \mathrm{~b}$ study. After a median follow-up period of 10.3 months, the study showed that the combination has an acceptable safety profile and an ORR of $62 \%(60)$. These results prompted the phase III trial (IMbrave150) to compare atezolizumab plus bevacizumab to single agent sorafenib in previously untreated patients with locally advanced or metastatic HCC (61). IMbrave150 is a global open-label phase III randomized trial that accrued 501 patients with advanced HCC and no prior systemic therapy who were randomized in a 2:1 ratio to atezolizumab $1,200 \mathrm{mg}$ and bevacizumab $15 \mathrm{mg} / \mathrm{Kg}$ administered intravenously every 3 weeks $v$ s. sorafenib $400 \mathrm{mg}$ orally twice daily. Eligible patients have confirmed HCC, have not received prior systemic therapy, Child Pugh liver function A, and ECOG performance status 
Table 1 Clinical trials of immunotherapies combinations in locally advanced unresectable and metastatic HCC

\begin{tabular}{|c|c|c|c|c|c|}
\hline Trial identifier & Line & Agents & Primary endpoint & Patients & Status \\
\hline NCT03298451 & First line & $\begin{array}{l}\text { Durvalumab vs. durvalumab + } \\
\text { tremelimumab vs. sorafenib }\end{array}$ & OS & 1,310 & Active, not recruiting \\
\hline NCT03412773 & First line & BGB-A317 (PD-1 antibody) vs. sorafenib & os & 674 & Active, not recruiting \\
\hline NCT03434379 & First line & $\begin{array}{c}\text { Atezolizumab + bevacizumab vs. } \\
\text { sorafenib }\end{array}$ & OS, PFS & 480 & Active, not recruiting \\
\hline NCT01658878 & First line & $\begin{array}{l}\text { Nivolumab + cabozantinib vs. nivolumab } \\
+ \text { ipilimumab + cabozantinib }\end{array}$ & $\begin{array}{l}\text { Safety, tolerability } \\
\text { and ORR }\end{array}$ & 1,097 & Active, not recruiting \\
\hline
\end{tabular}

PFS, progression free survival; OS, overall survival; TTP, time to progression; TR-RECIST, tumor response using Response Evaluation Criteria in Solid Tumors (RECIST); ORR, objective response rate; TEAEs, treatment-emergent adverse events; DLTs, dose limiting toxicities; MTD, maximum tolerated dose.

of 0 or 1 . Patients must undergo screening and adequate treatment of esophageal varices before initiating therapy. The combination of atezolizumab plus bevacizumab showed improvement in OS and PFS, the two coprimary endpoints of the study, compared to sorafenib. After a median follow-up of 8.6 months at the time of study report, median OS for sorafenib was 13.2 months and not yet reached for the combination, HR was 0.58 (95\% CI, 0.42-0.79; $\mathrm{P}<0.001)$. PFS was improved from 4.3 months for sorafenib to 6.8 months for atezolizumab and bevacizumab combination, HR, 0.59; 95\% CI, 0.47-0.76; $\mathrm{P}<0.001)$. the investigational arm ORR was $27.3 \%$ vs. $11.9 \%$ in sorafenib $(\mathrm{P}<0.001)$ using RECIST1.1 criteria. The difference was similarly noted and significant when using mRECIST. Patient-reported outcomes demonstrated a delayed time to deterioration of quality of life with atezolizumab-bevacizumab combination (11.2 months) compared to sorafenib (3.2 months), HR $0.63 ; 95 \%$ CI, 0.46 to 0.85 . These breakthrough results can potentially be practice changing and lead to a new first line treatment approach for advanced unresectable HCC. This was the first study to show superiority over sorafenib and the first positive frontline checkpoint inhibitor based study.

\section{TKI and checkpoint inbibitor}

Similarly, the combination of TKI and immunotherapy was studied in different solid tumors. Sorafenib, lenvatinib, and cabozantinib exert immune modulation effect as shown in preclinical and clinical data $(62,63)$, making combining these agents with checkpoint blockade an attractive approach in HCC as well.

\section{Pembrolizumab and lenvatinib}

Pembrolizumab and lenvatinib combination was studied in the KEYNOTE-524 open label phase $1 \mathrm{~b}$ trial. The trial was initially designed to assess for dose limiting toxicities which none of the enrolled patients manifested. Additional patients were enrolled to second part of the study which evaluated safety and tolerability as primary outcomes and efficacy (ORR and PFS) using modified RECIST (mRECIST) as secondary outcomes. The combination therapy was deemed to have a favorable safety profile and a durable ORR of $44.8 \%$ with a DOR of 18.7 months (64). In light of these promising results an ongoing phase III trial (LEAP-002) is testing the use of pembrolizumab and lenvatinib combination compared to lenvatinib alone as frontline treatment in patients with advanced HCC (65).

\section{Combinations of checkpoint inbibitors}

Targeting different pathways of the immune checkpoint at the same time improved outcomes in melanoma (66) and this approach is being evaluated in HCC. 


\section{Ipilimumab and nivolumab}

CHECKMATE 040 discussed above, had an arm extension evaluating the combination of PD-1 and CTLA-4 inhibition in sorafenib-treated HCC patients. Patients received nivolumab $3 \mathrm{mg} / \mathrm{kg}$ and ipilimumab $1 \mathrm{mg} / \mathrm{kg}$ for 4 doses. Patients randomized to the combination arm had an overall response rate of $31 \%$. Interestingly, complete response was reported in 7 patients. 24 -months OS was $40 \%$, with an acceptable safety profile (67).

\section{Durvalumab and tremelimumab}

Preclinical data has shown that blocking both PD-L1 and anti-CTLA-4 pathways enhanced anti-tumor activity (68). Early results from a phase I/II trial combining anti-PD-L1 and anti-CTLA-4 antibodies established acceptable toxicity profile and an ORR of 18\% (NCT02519348) (69). A more recent data cut-off update was recently released (70). Three Hundred thirty-two patients were enrolled. Four regimens were evaluated, durvalumab (D) and tremelimumab (T) as monotherapy, and two different dosing of the two drugs combination ( $\mathrm{T} 300 \mathrm{mg} / \mathrm{D}$ for one dose followed by D every 4 weeks vs. T $75 \mathrm{mg} / \mathrm{D}$ every 4 weeks for 4 doses followed by D every 4 weeks). The highest observed ORR was for T 300/D combination, at 22.7\%. Median OS was 18.7 months (10.8-not reached) for T 300/D, 11.3 months (8.4-14.6) for T75/D combination, 11.7 months (8.5-16.9) for D monotherapy, and 17.1 months (10.9-not reached) for $\mathrm{T}$ monotherapy, given at $750 \mathrm{mg}$ every 4 weeks. Grade 3 or 4 treatment related adverse events were $35.1 \%, 24.4 \%$, $17.8 \%$, and $42 \%$, for each of the study arms, respectively.

A phase III open-label, multicenter, randomized study is evaluating the combination of CTLA-4 and PD-1 checkpoint blockade in HCC (HIMALAYA) ClinicalTrials. gov Identifier: NCT03298451. Patients are randomized to receive durvalumab monotherapy versus durvalumab and tremelimumab combination, versus sorafenib (71). The study is looking at OS as primary endpoint, PFS and ORR as secondary endpoints. The study aimed to enroll 1,310 patients across the world.

\section{Triplet combination}

\section{Ipilimumab and nivolumab with cabozantinib}

A subanalysis of the CheckMate 040, the multi-arm phase I/II study, tested the efficacy of ipilimumab in combination with nivolumab and cabozantinib in both sorafenib- naïve and sorafenib treated advanced HCC compared to the combination of nivolumab and cabozantinib alone. Preliminary results demonstrated an ORR by RECIST v1.1 which was the primary endpoint, of $26 \%$ in the ipilimumab plus combination group vs. $17 \%$ in the combination nivolumab and cabozantinib alone. Grade 3-4 treatment related adverse events were more common in patients receiving the triplet therapy (71\%) compared to those receiving the combination therapy (42\%). An improvement in median PFS of 6.8 months (95\% CI, 4.0-14.3) compared to 5.5 months (95\% CI, 3.2-10.9) was perceived (72). Of course, these findings would be interpreted within the context of a subset analysis of the phase 2 study.

\section{Systemic-regional therapy combination}

Recent interest is rising in combining liver-directed approach with the novel therapeutics. Sorafenib has been tested as adjuvant therapy post liver resection (73) and extensively in combination with locoregional therapies such as transarterial chemoembolization (TACE). A prospective phase 2 study of 35 patients initially established safety of combining sorafenib with TACE (74). Subsequently, several randomized trials were conducted (75-78) with no substantial benefit in survival outcomes.

The SPACE trial, a randomized, double-blind, phase II trial, tested the safety as well as efficacy of drug-eluting beads (deb)-TACE with or without sorafenib in patients with intermediate stage HCC. A total of 307 patients were randomized to undergo deb-TACE plus sorafenib or placebo with the primary endpoint being time to tumor progression (TTP). There was no significant difference in median TTP between both study groups. However, the trial did demonstrate a lower time-to-"unTACEable" progression in the deb-TACE plus sorafenib group with a HR 1.586 (95\% CI, 1.200-2.096), a secondary endpoint.

Similarly, TACE 2 trial, a randomized placebocontrolled, double-blind, multicenter phase 3 trial also tested the use of deb-TACE with or without sorafenib in patients with unresectable liver-confined HCC (78). The primary endpoint was PFS. The addition of sorafenib to deb-TACE did not improve PFS compared to deb-TACE + placebo in patient with intermediate stage HCC with a HR of 0.99 and $\mathrm{P}=0.94$.

A randomized, double-blind, phase III trial, ECOG 1208 (NCT01004978) addressed the same question in unresectable HCC, with and without vascular invasion. Patients received TACE (with either doxorubicin 
Table 2 Ongoing clinical trials of combination immunotherapy and locoregional treatment

\begin{tabular}{lccc}
\hline Trial identifier & Agent/regional therapy & Primary endpoint & Status \\
\hline NCT03143270 & Nivolumab with deb-TACE & Safety & Ongoing, recruiting \\
NCT03099564 & Pembrolizumab with Y-90 radioembolization & PFS & Ongoing, recruiting \\
NCT03482102 & Durvalumab, tremelimumab radiation therapy & ORR & Ongoing, recruiting \\
\hline
\end{tabular}

PFS, progression free survival; ORR, objective response rate; deb-TACE, drug-eluting bead transarterial chemoembolization.

hydrochloride, mitomycin $\mathrm{C}$, cisplatin, or doxorubicineluting beads) with sorafenib or placebo. The primary endpoint was to compare PFS difference between study arms, regardless of area of progression, while the secondary endpoints included OS, safety, and patterns of liver failure whether extra-hepatic or intra-hepatic. The study was held for futility.

In contrast, the TACTICS trial, a multicenter phase II trial, explored the use of sorafenib in combination with TACE versus TACE alone in unresectable HCC; 156 patients were randomized to receive TACE alone or TACE plus sorafenib. Interestingly, primary endpoint was PFS defined by "time to unTACEable progression", as opposed to conventional PFS. Intrahepatic new lesion was not considered tumor progression. The addition of sorafenib to TACE translated into improvement in median PFS compared to TACE only; 25.2 vs. 13.5 months, $\mathrm{P}=0.006$. Furthermore, the trial demonstrated an increase in time to untreatable progression in the sorafenib group of $26.7 v$ s. 20.6 months in patients receiving TACE alone with a $\mathrm{P}$ value of 0.02 . The $\mathrm{OS}$ was not evaluable as the number for events were not reached. The difference in outcomes between TACTICS and other similar reported studies could be attributed to difference in radiological criteria, longer use of sorafenib, and patient's selection. Nonetheless, the difference may be explained in the trial design, where PFS was denied as progression within treated area not amenable for further TACE. Furthermore, sorafenib was administered few weeks before TACE, which might have caused delay in treatment start in the control arm and the worse outcome.

More recently, locoregional therapies such as embolization, ablation, and radiation, are being studied in combination with checkpoint inhibition. Earlier a study of tremelimumab and ablation in 32 patients with advanced HCC (BCLC stage C and must have had progressed on or been not tolerant to prior sorafenib). Partial response was $26.3 \%$ (95\% CI, 9.1-51.2\%), biopsies at 6-week from treatment showed an increase in CD8+ T cells. Median
TTP was 7.4 months and median OS reached 12.3 months. These results suggest that direct cancer cells killing can activate the immune system paving the way for immune checkpoint inhibitors (79).

\section{Nivolumab with deb-TACE}

An ongoing multicenter pilot phase I/II study is testing the use of nivolumab in combination with deb-TACE loaded with doxorubicin in patients with unresectable HCC (BCLC B). The primary endpoint of the study is tolerability, safety, and to identify the optimal dosing schedule of the combination (80). Preliminary results were reported recently in abstract form. Out of the 9 patients treated so far none experienced treatment related liver failure, dose-limiting toxicity, or Grade 5 adverse events (NCT03143270) (81).

Currently, several agents are being evaluated as single agent monotherapy or in combination along with various loco-regional therapies such as TACE and radioembolization (Table 2).

\section{Interferon (IFN) therapy}

Historically, IFN were broadly studied in HCC, both in the adjuvant and advanced setting, with overall no consensus about efficacy as benefit was not reproduced in randomized studies and a large concern on toxicity $(82,83)$. TGF $\beta$ signaling has been associated with HCC (84). A single arm phase 2 study of galunisertib, a TGF $\beta$ inhibitor, and sorafenib in patients with advanced HCC was completed. Preliminary results on 47 enrolled patients were reported in abstract form. The combination showed a favorable safety profile and a median OS of 17.9 months (85). At mature follow-up TTP was 4.1 months and median OS was 18.8 months (95\% CI, 14.8, 24.8). partial response was observed in $4.5 \%$ of patients per RECIST1.1 criteria (86). Interestingly, TGF- $\beta 1$ responders (as defined by decrease of $>20 \%$ from baseline) $v s$. non-responders had longer OS 
Page 8 of 12

(22.8 vs. 12.0 months, $\mathrm{P}=0.038)$.

\section{Cellular therapy}

Chimeric antigen receptor (CAR) engineered T-cell therapy is currently approved in many hematological malignancies and seems to be on the horizon in liver tumors. Preclinical studies have evaluated adoptive glypican-3 (GPC3) CAR T therapy in patient-derived xenograft (PDX) models. The treatment was able to slow down tumor growth and eradicate tumor cells in which GPC3 was highly expressed (87). Early phase clinical trials are undergoing to test different CAR targets in patients with HCC (NCT02905188, NCT03884751, NCT03980288, NCT03993743). In addition to GPC3, other potential targets being looked at include AFP, human telomerase reverse transcriptase (hTERT), and melanoma antigen gene (MAGE3). Although AFP as a tumor associated epitope is frequently secreted in HCC, its specific immunological responses has not been well understood in HCC and the right APC human leucocyte antigen (HLA) is yet to be identified. AFP-derived peptides containing HLA-A*2402 binding motifs that showed high binding affinity to HLA-A*2402 induced cytotoxic T cells to produce IFN-gamma and kill an AFP-producing hepatoma cell line (88). A fully human AFP CAR T cell complexed with HLA-A*02:01 was tested in mouse models and demonstrated antitumor activity (89). This approach is at the early stages of exploration, mature data and clinical experience is surely needed.

\section{Conclusions}

As delineated in this systematic review, the treatment paradigm in HCC has shifted dramatically over the last few years, and we are witnessing a rapid influx of data changing our way treating patients with advanced HCC at an unprecedented pace.

TKIs, immunotherapy, and the combination of both are all efficient therapies showing meaningful improvement in outcomes.

Certain questions pertinent to use of these therapies remain unanswered. These include but are not limited to sequencing of the different options. This may be difficult to ascertain while we remain the current dynamic state of rapidly evolving data. Personalizing therapies based on etiology and molecular sequencing among other variables would be key.
El Dika et al. Novel systemic therapies in hepatocellular carcinoma

\section{Acknowledgments}

Funding: None.

\section{Footnote}

Provenance and Peer Review: This article was commissioned by the Guest Editors (Mehmet Akce and Shishir K. Maithel) for the series "Hepatocellular Carcinoma" published in Chinese Clinical Oncology. The article was sent for external peer review organized by the Guest Editors and the editorial office.

Conflicts of Interest: All authors have completed the ICMJE uniform disclosure form (available at http://dx.doi. org/10.21037/cco-20-113). The series "Hepatocellular Carcinoma" was commissioned by the editorial office without any funding or sponsorship. GKAA serves as an unpaid associate editor-in-chief of Chinese Clinical Oncology from October 2017 to September 2022. GKAA reports grants from ActaBiologica, Agios, Array, Astra Zeneca, Bayer, Beigene, BMS, Casi, Celgene, Exelixis, Genentech, Halozyme, Incyte, Mabvax, Polaris Puma, QED, Roche, personal fees from Agios, Astra Zeneca, Autem, Bayer, Beigene, Berry Genomics, Celgene, CytomX, Debio, Eisai, Eli Lilly, Flatiron, Genentech, Gilead, Incyte, Ipsen, LAM, Loxo, Merck, MINA, QED, Redhill, Roche, Silenseed, Sillajen, Sobi, Therabionics, Twoxar, Vector, Yiviva, outside the submitted work. In addition, GKAA has a patent ARTICLES AND METHODS FOR PREVENTING AND TREATING DERMATOLOGIC ADVERSE EVENTS, identified by International Patent Application No. PCT/US2014/031545 filed on March 24, 2014, and priority application Serial No.: 61/804,907; Filed: March 25,2013 issued. The other authors have no other conflicts of interest to declare.

Ethical Statement: The authors are accountable for all aspects of the work in ensuring that questions related to the accuracy or integrity of any part of the work are appropriately investigated and resolved.

Open Access Statement: This is an Open Access article distributed in accordance with the Creative Commons Attribution-NonCommercial-NoDerivs 4.0 International License (CC BY-NC-ND 4.0), which permits the noncommercial replication and distribution of the article with the strict proviso that no changes or edits are made and the 
original work is properly cited (including links to both the formal publication through the relevant DOI and the license). See: https://creativecommons.org/licenses/by-nc-nd/4.0/.

\section{References}

1. Zhou M, Wang H, Zeng X, et al. Mortality, Morbidity, and Risk Factors in China and Its Provinces, 1990-2017: A Systematic Analysis for the Global Burden of Disease Study 2017. Lancet 2019;394:1145-58.

2. Bray F, Ferlay J, Soerjomataram I, et al. Global cancer statistics 2018: GLOBOCAN estimates of incidence and mortality worldwide for 36 cancers in 185 countries. CA Cancer J Clin 2018;68:394-424.

3. Baecker A, Liu X, La Vecchia C, et al. Worldwide incidence of hepatocellular carcinoma cases attributable to major risk factors. Eur J Cancer Prev 2018;27:205-12.

4. Sayiner M, Golabi P, Younossi ZM. Disease Burden of Hepatocellular Carcinoma: A Global Perspective. Dig Dis Sci 2019;64:910-7.

5. Altekruse SF, McGlynn KA, Reichman ME. Hepatocellular carcinoma incidence, mortality, and survival trends in the United States from 1975 to 2005. J Clin Oncol 2009;27:1485.

6. Petrick JL, Kelly SP, Altekruse SF, et al. Future of Hepatocellular Carcinoma Incidence in the United States Forecast Through 2030. J Clin Oncol 2016;34:1787-94.

7. Klevens RM, Liu S, Roberts H, et al. Estimating acute viral hepatitis infections from nationally reported cases. Am J Public Health 2014;104:482-7.

8. Younossi ZM, Koenig AB, Abdelatif D, et al. Global epidemiology of nonalcoholic fatty liver disease-metaanalytic assessment of prevalence, incidence, and outcomes. Hepatology 2016;64:73-84.

9. European Association For The Study Of The Liver; European Organisation For Research And Treatment Of Cancer. EASL-EORTC clinical practice guidelines: management of hepatocellular carcinoma. J Hepatol 2012;56:908-43.

10. Morris-Stiff G, Gomez D, de Liguori Carino N, et al. Surgical management of hepatocellular carcinoma: is the jury still out? Surg Oncol 2009;18:298-321.

11. Imamura H, Matsuyama Y, Tanaka E, et al. Risk factors contributing to early and late phase intrahepatic recurrence of hepatocellular carcinoma after hepatectomy. J Hepatol 2003;38:200-7.

12. Zheng J, Chou JF, Gonen M, et al. Prediction of Hepatocellular Carcinoma Recurrence Beyond Milan
Criteria After Resection: Validation of a Clinical Risk Score in an International Cohort. Ann Surg 2017;266:693-701.

13. Famularo S, Di Sandro S, Giani A, et al. Recurrence Patterns After Anatomic or Parenchyma-Sparing Liver Resection for Hepatocarcinoma in a Western Population of Cirrhotic Patients. Ann Surg Oncol 2018;25:3974-81.

14. Lin S, Hoffmann K, Schemmer P. Treatment of hepatocellular carcinoma: a systematic review. Liver cancer 2012;1:144-58.

15. Llovet JM, Ricci S, Mazzaferro V, et al. Sorafenib in advanced hepatocellular carcinoma. N Engl J Med 2008;359:378-90.

16. Rimassa L, Santoro A. Sorafenib therapy in advanced hepatocellular carcinoma: the SHARP trial. Expert Rev Anticancer Ther 2009;9:739-45.

17. Cheng AL, Kang YK, Chen Z, et al. Efficacy and safety of sorafenib in patients in the Asia-Pacific region with advanced hepatocellular carcinoma: a phase III randomised, double-blind, placebo-controlled trial. Lancet Oncol 2009;10:25-34.

18. Cheng AL, Kang YK, Lin DY, et al. Sunitinib versus sorafenib in advanced hepatocellular cancer: results of a randomized phase III trial. J Clin Oncol 2013;31:4067-75.

19. Cainap C, Qin S, Huang WT, et al. Linifanib versus Sorafenib in patients with advanced hepatocellular carcinoma: results of a randomized phase III trial. J Clin Oncol 2015;33:172.

20. Koeberle D, Dufour JF, Demeter G, et al. Sorafenib with or without everolimus in patients with advanced hepatocellular carcinoma (HCC): a randomized multicenter, multinational phase II trial (SAKK 77/08 and SASL 29). Ann Oncol 2016;27:856-61.

21. Yamamoto Y, Matsui J, Matsushima T, et al. Lenvatinib, an angiogenesis inhibitor targeting VEGFR/FGFR, shows broad antitumor activity in human tumor xenograft models associated with microvessel density and pericyte coverage. Vasc Cell 2014;6:18.

22. Kudo M, Finn RS, Qin S, et al. Lenvatinib versus sorafenib in first-line treatment of patients with unresectable hepatocellular carcinoma: a randomised phase 3 noninferiority trial. Lancet 2018;391:1163-73.

23. Bruix J, Qin S, Merle P, et al. Regorafenib for patients with hepatocellular carcinoma who progressed on sorafenib treatment (RESORCE): a randomised, double-blind, placebo-controlled, phase 3 trial. Lancet 2017;389:56-66.

24. Alsina A, Kudo M, Vogel A, et al. Effects of Subsequent Systemic Anticancer Medication Following First-Line 


\section{Page 10 of 12}

Lenvatinib: A Post Hoc Responder Analysis from the Phase 3 REFLECT Study in Unresectable Hepatocellular Carcinoma. Liver Cancer 2020;9:93-104.

25. Finn RS, Merle P, Granito A, et al. Outcomes of sequential treatment with sorafenib followed by regorafenib for HCC: Additional analyses from the phase III RESORCE trial. J Hepatol 2018;69:353-8.

26. Xiang Q, Chen W, Ren M, et al. Cabozantinib Suppresses Tumor Growth and Metastasis in Hepatocellular Carcinoma by a Dual Blockade of VEGFR2 and MET. Clin Cancer Res 2014;20:2959-70.

27. Abou-Alfa GK, Meyer T, Cheng AL, et al. Cabozantinib in Patients with Advanced and Progressing Hepatocellular Carcinoma. N Engl J Med 2018;379:54-63.

28. Abou-Alfa GK, Meyer T, Cheng AL, et al. Association of adverse events (AEs) with efficacy outcomes for cabozantinib (C) in patients (pts) with advanced hepatocellular carcinoma (aHCC) in the phase III CELESTIAL trial. J Clin Oncol 2019;37:4088.

29. Rimassa L, Cicin I, Blanc JF, et al. Outcomes based on age in the phase 3 CELESTIAL trial of cabozantinib (C) versus placebo $(\mathrm{P})$ in patients (pts) with advanced hepatocellular carcinoma (HCC). J Clin Oncol 2018;36:4090.

30. Miksad R, Cicin I, Chen Y, et al. O-022 Outcomes based on Albumin-Bilirubin (ALBI) grade in the phase 3 CELESTIAL trial of cabozantinib versus placebo in patients with advanced hepatocellular carcinoma (HCC). Ann Oncol 2019;30:mdz154.021.

31. Johnson PJ, Berhane S, Kagebayashi C, et al. Assessment of liver function in patients with hepatocellular carcinoma: a new evidence-based approach-the ALBI grade. J Clin Oncol 2015;33:550-8.

32. Lee SK, Song MJ, Kim SH, et al. Comparing various scoring system for predicting overall survival according to treatment modalities in hepatocellular carcinoma focused on Platelet-albumin-bilirubin (PALBI) and albuminbilirubin (ALBI) grade: A nationwide cohort study. PLoS One 2019;14:e216173.

33. Edeline J, Blanc JF, Johnson P, et al. A multicentre comparison between Child Pugh and Albumin-Bilirubin scores in patients treated with sorafenib for Hepatocellular Carcinoma. Liver Int 2016;36:1821-8.

34. Ho CHM, Chiang CL, Lee FAS, et al. Comparison of platelet-albumin-bilirubin (PALBI), albumin-bilirubin (ALBI), and child-pugh (CP) score for predicting of survival in advanced hcc patients receiving radiotherapy (RT). Oncotarget 2018;9:28818-29.

\section{El Dika et al. Novel systemic therapies in hepatocellular carcinoma}

35. Pazo Cid RA, Esquerdo G, Puertolas T, et al. Bevacizumab (BVZ) as second-line treatment after sorafenib (SFB) progression in patients (pts) with advanced hepatocellular carcinoma (HCC). J Clin Oncol 2010;28:e14619.

36. Hubbard JM, Mahoney MR, Loui WS, et al. Phase I/ II Randomized Trial of Sorafenib and Bevacizumab as First-Line Therapy in Patients with Locally Advanced or Metastatic Hepatocellular Carcinoma: North Central Cancer Treatment Group Trial N0745 (Alliance). Target Oncol 2017;12:201-9.

37. Zhu AX, Park JO, Ryoo BY, et al. Ramucirumab versus placebo as second-line treatment in patients with advanced hepatocellular carcinoma following first-line therapy with sorafenib (REACH): a randomised, double-blind, multicentre, phase 3 trial. Lancet Oncol 2015;16:859-70.

38. Zhu AX, Kang YK, Yen CJ, et al. Ramucirumab after sorafenib in patients with advanced hepatocellular carcinoma and increased alpha-fetoprotein concentrations (REACH-2): a randomised, double-blind, placebocontrolled, phase 3 trial. Lancet Oncol 2019;20:282-96.

39. Thomson AW, Knolle PA. Antigen-presenting cell function in the tolerogenic liver environment. Nat Rev Immunol 2010;10:753-66.

40. Harding JJ, El Dika I, Abou-Alfa GK. Immunotherapy in hepatocellular carcinoma: Primed to make a difference? Cancer 2016;122:367-77.

41. Kuang DM, Zhao Q, Peng C, et al. Activated monocytes in peritumoral stroma of hepatocellular carcinoma foster immune privilege and disease progression through PD-L1. J Exp Med 2009;206:1327-37.

42. Zeng Z, Shi F, Zhou L, et al. Upregulation of circulating PD-L1/PD-1 is associated with poor post-cryoablation prognosis in patients with HBV-related hepatocellular carcinoma. PLoS One 2011;6:e23621.

43. Khalil DN, Smith EL, Brentjens RJ, et al. The future of cancer treatment: immunomodulation, CARs and combination immunotherapy. Nat Rev Clin Oncol 2016;13:273-90.

44. Gao Q, Qiu SJ, Fan J, et al. Intratumoral balance of regulatory and cytotoxic $\mathrm{T}$ cells is associated with prognosis of hepatocellular carcinoma after resection. J Clin Oncol 2007;25:2586-93.

45. Bhardwaj N, Li M, Price T, et al. Spontaneous regression of a biopsy confirmed hepatocellular carcinoma. BMJ Case Rep 2014;2014:bcr2014204897.

46. El-Khoueiry AB, Sangro B, Yau T, et al. Nivolumab in patients with advanced hepatocellular carcinoma (CheckMate 040): an open-label, non-comparative, phase $1 / 2$ dose escalation and expansion trial. Lancet 
2017;389:2492-502.

47. Yau T, Park J, Finn R, et al. CheckMate 459: A randomized, multi-center phase III study of nivolumab (NIVO) vs sorafenib (SOR) as first-line (1L) treatment in patients (pts) with advanced hepatocellular carcinoma (aHCC). Ann Oncol 2019;30:v874-5.

48. Zhu AX, Finn RS, Cattan S, et al. KEYNOTE-224: Pembrolizumab in patients with advanced hepatocellular carcinoma previously treated with sorafenib. J Clin Oncol 2018;36;4_suppl:209.

49. Finn RS, Ryoo BY, Merle P, et al. Results of KEYNOTE-240: phase 3 study of pembrolizumab (Pembro) vs best supportive care (BSC) for second line therapy in advanced hepatocellular carcinoma (HCC). J Clin Oncol 2019;37;15_suppl:4004.

50. Abou-Alfa GK, Venook AP. The antiangiogenic ceiling in hepatocellular carcinoma: does it exist and has it been reached? Lancet Oncol 2013;14:e283-8.

51. Kimura T, Kato Y, Ozawa Y, et al. Immunomodulatory activity of lenvatinib contributes to antitumor activity in the Hepa1-6 hepatocellular carcinoma model. Cancer Sci 2018;109:3993-4002.

52. Noman MZ, Desantis G, Janji B, et al. PD-L1 is a novel direct target of HIF-1 $\alpha$, and its blockade under hypoxia enhanced MDSC-mediated T cell activation. J Exp Med 2014;211:781-90.

53. Terme M, Pernot S, Marcheteau E, et al. VEGFAVEGFR pathway blockade inhibits tumor-induced regulatory T-cell proliferation in colorectal cancer. Cancer Res 2013;73:539-49.

54. Liu XD, Hoang A, Zhou L, et al. Resistance to Antiangiogenic Therapy Is Associated with an Immunosuppressive Tumor Microenvironment in Metastatic Renal Cell Carcinoma. Cancer Immunol Res 2015;3:1017-29.

55. Hellwig SM, Damen CA, van Adrichem NP, et al. Endothelial CD34 is suppressed in human malignancies: role of angiogenic factors. Cancer Lett 1997;120:203-11.

56. Reck M, Mok TSK, Nishio M, et al. Atezolizumab plus bevacizumab and chemotherapy in non-small-cell lung cancer (IMpower150): key subgroup analyses of patients with EGFR mutations or baseline liver metastases in a randomised, open-label phase 3 trial. Lancet Respir Med 2019;7:387-401.

57. Rini BI, Powles T, Atkins MB, et al. Atezolizumab plus bevacizumab versus sunitinib in patients with previously untreated metastatic renal cell carcinoma (IMmotion151): a multicentre, open-label, phase 3 , randomised controlled trial. Lancet 2019;393:2404-15.

58. Ohm JE, Carbone DP. VEGF as a mediator of tumor-associated immunodeficiency. Immunol Res 2001;23:263-72.

59. Manegold C, Dingemans AC, Gray JE, et al. The Potential of Combined Immunotherapy and Antiangiogenesis for the Synergistic Treatment of Advanced NSCLC. J Thorac Oncol 2017;12:194-207.

60. Stein S, Pishvaian MJ, Lee MS, et al. Safety and clinical activity of $1 \mathrm{~L}$ atezolizumab + bevacizumab in a phase $\mathrm{Ib}$ study in hepatocellular carcinoma (HCC). J Clin Oncol 2018;36:4074.

61. Finn RS, Qin S, Ikeda M, et al. Atezolizumab plus Bevacizumab in Unresectable Hepatocellular Carcinoma. N Engl J Med 2020;382:1894-905.

62. Ribas A, Wolchok JD. Combining cancer immunotherapy and targeted therapy. Curr Opin Immunol 2013;25:291-6.

63. Cabrera R, Ararat M, Xu Y, et al. Immune modulation of effector CD4+ and regulatory $\mathrm{T}$ cell function by sorafenib in patients with hepatocellular carcinoma. Cancer Immunol Immunother 2013;62:737-46.

64. Llovet J, Shepard K, Finn R, et al. A phase Ib trial of lenvatinib (LEN) plus pembrolizumab (PEMBRO) in unresectable hepatocellular carcinoma (uHCC): Updated results. Ann Oncol 2019;30:v286-7.

65. Llovet JM, Kudo M, Cheng AL, et al. Lenvatinib (len) plus pembrolizumab (pembro) for the first-line treatment of patients (pts) with advanced hepatocellular carcinoma (HCC): Phase 3 LEAP-002 study. J Clin Oncol 2019. doi: 10.1200/JCO.2019.37.15_suppl.TPS4152.

66. Hodi FS, Chesney J, Pavlick AC, et al. Combined nivolumab and ipilimumab versus ipilimumab alone in patients with advanced melanoma: 2-year overall survival outcomes in a multicentre, randomised, controlled, phase 2 trial. Lancet Oncol 2016;17:1558-68.

67. Yau T, Kang YK, Kim TY, et al. Nivolumab (NIVO) + ipilimumab (IPI) combination therapy in patients (pts) with advanced hepatocellular carcinoma (aHCC): Results from CheckMate 040. J Clin Oncol 2019;37:4012.

68. Stewart R, Mullins S, Watkins A, et al. Preclinical modelling of immune checkpoint blockade (P2012). J Immunol 2013;190:214.7.

69. Kelley RK, Abou-Alfa GK, Bendell JC, et al. Phase I/II study of durvalumab and tremelimumab in patients with unresectable hepatocellular carcinoma (HCC): Phase I safety and efficacy analyses. J Clin Oncol 2017;35:4073.

70. Kelly R. Efficacy, tolerability, and biologic activity of a novel regimen of tremelimumab $(\mathrm{T})$ in combination 
with durvalumab (D) for patients (pts) with advanced hepatocellular carcinoma (aHCC). J Clin Oncol 2020;38:abstr 4508.

71. Abou-Alfa GK, Chan SL, Furuse J, et al. A randomized, multicenter phase 3 study of durvalumab (D) and tremelimumab $(\mathrm{T})$ as first-line treatment in patients with unresectable hepatocellular carcinoma (HCC): HIMALAYA study. J Clin Oncol 2018;36:TPS4144.

72. Yau T. Nivolumab (NIVO) + ipilimumab (IPI) + cabozantinib (CABO) combination therapy in patients (pts) with advanced hepatocellular carcinoma (aHCC): Results from CheckMate 040. GI Cancers Symposium; January 23-25, 2020; San Francisco, CA, 2019.

73. Bruix J, Takayama T, Mazzaferro V, et al. Adjuvant sorafenib for hepatocellular carcinoma after resection or ablation (STORM): a phase 3, randomised, double-blind, placebo-controlled trial. Lancet Oncol 2015;16:1344-54.

74. Pawlik TM, Reyes DK, Cosgrove D, et al. Phase II trial of sorafenib combined with concurrent transarterial chemoembolization with drug-eluting beads for hepatocellular carcinoma. J Clin Oncol 2011;29:3960-7.

75. Liu L, Chen H, Wang M, et al. Combination therapy of sorafenib and TACE for unresectable HCC: a systematic review and meta-analysis. PLoS One 2014;9:e91124.

76. Kudo M, Ueshima K, Ikeda M, et al. Randomized, open label, multicenter, phase II trial comparing transarterial chemoembolization (TACE) plus sorafenib with TACE alone in patients with hepatocellular carcinoma (HCC): TACTICS trial. J Clin Oncol 2018;36:206.

77. Lencioni R, Llovet JM, Han G, et al. Sorafenib or placebo plus TACE with doxorubicin-eluting beads for intermediate stage HCC: The SPACE trial. J Hepatol 2016;64:1090-8.

78. Meyer T, Fox R, Ma YT, et al. Sorafenib in combination with transarterial chemoembolisation in patients with unresectable hepatocellular carcinoma (TACE 2): a randomised placebo-controlled, double-blind, phase 3 trial. Lancet Gastroenterol Hepatol 2017;2:565-75.

79. Duffy AG, Ulahannan SV, Makorova-Rusher O, et al. Tremelimumab in combination with ablation in patients with advanced hepatocellular carcinoma. J Hepatol 2017;66:545-51.

80. Harding JJ, Erinjeri JP, Tan BR, et al. A multicenter pilot study of nivolumab (NIVO) with drug eluting bead transarterial chemoembolization (deb-TACE) in patients (pts) with liver limited hepatocellular carcinoma (HCC). J Clin Oncol 2018. doi: 10.1200/JCO.2018.36.15_suppl. TPS4146.

81. Harding JJ, Yarmohammadi H, Reiss KA, et al. Nivolumab (NIVO) and drug eluting bead transarterial chemoembolization (deb-TACE): Preliminary results from a phase I study of patients (pts) with liver limited hepatocellular carcinoma (HCC). J Clin Oncol 2020;38:525.

82. Llovet JM, Sala M, Castells L, et al. Randomized controlled trial of interferon treatment for advanced hepatocellular carcinoma. Hepatology 2000;31:54-8.

83. Mazzaferro V, Romito R, Schiavo M, et al. Prevention of hepatocellular carcinoma recurrence with alpha-interferon after liver resection in $\mathrm{HCV}$ cirrhosis. Hepatology 2006;44:1543-54.

84. Liu J, Chen S, Wang W, et al. Cancer-associated fibroblasts promote hepatocellular carcinoma metastasis through chemokine-activated hedgehog and TGF-beta pathways. Cancer Lett 2016;379:49-59.

85. Kelley RK, Gane E, Assenat E, et al. A phase 2 study of galunisertib (TGF-B R1 inhibitor) and sorafenib in patients with advanced hepatocellular carcinoma (HCC). J Clin Oncol 2017;35:4097.

86. Kelley RK, Gane E, Assenat E, et al. A Phase 2 Study of Galunisertib (TGF-beta1 Receptor Type I Inhibitor) and Sorafenib in Patients With Advanced Hepatocellular Carcinoma. Clin Transl Gastroenterol 2019;10:e0056.

87. Jiang Z, Jiang X, Chen S, et al. Anti-GPC3-CAR T Cells Suppress the Growth of Tumor Cells in Patient-Derived Xenografts of Hepatocellular Carcinoma. Front Immunol 2017;7:690.

88. Mizukoshi E, Nakamoto Y, Tsuji H, et al. Identification of alpha-fetoprotein-derived peptides recognized by cytotoxic T lymphocytes in HLA-A24+ patients with hepatocellular carcinoma. Int J Cancer 2006;118:1 194-204.

89. Liu H, Xu Y, Xiang J, et al. Targeting Alpha-Fetoprotein (AFP)-MHC Complex with CAR T-Cell Therapy for Liver Cancer. Clin Cancer Res 2017;23:478-88.

Cite this article as: El Dika I, Makki I, Abou-Alfa GK. Hepatocellular carcinoma, novel therapies on the horizon. Chin Clin Oncol 2021;10(1):12. doi: 10.21037/cco-20-113 